\title{
Meshless solution of laminar flow in a 2D backward-facing step
}

\author{
A. G. Vidal ${ }^{1}$, E. A. Divo ${ }^{2}$ \& A. J. Kassab ${ }^{1}$ \\ ${ }^{I}$ Department of Mechanical and Aerospace Engineering, \\ University of Central Florida, USA \\ ${ }^{2}$ Department of Mechanical Engineering, \\ Embry-Riddle Aeronautical University, USA
}

\begin{abstract}
A meshless procedure for 2D incompressible laminar flows is presented. The velocity-pressure coupling is done with the flow equations in their original form and no approximations or simplifications are done in the boundary conditions. Additionally, all needed interpolations are done with the effective Radial-Based Functions (RBF). The method is tested with the 2D backward-facing step and this new procedure predicts correctly the transition stage, in which the reattachment decreases and stabilizes as the flow enters in the turbulent regime.
\end{abstract} Keywords: meshless, CFD, incompressible flow, backward-facing step.

\section{Introduction}

Since the introduction of the Projection Method by Harlow and Welch [1], the science of Computational Fluid Dynamics (CFD) has become a fundamental tool for engineering calculations and design. Basically, the velocity-pressure coupling is done in a segregated way, one equation at a time. After the publication of this procedure, almost all numerical methods developed in Computational Fluid Dynamics (CFD), with some minor modifications, the original flow equations are transformed into a series of consecutive and explicit equations for velocity, pressure and mass correction, this last one needed to satisfy the mass balance. In spite of the improvement in all CFD techniques, even today the solution of complex elliptic problems, such as the backward-facing step or lid-driven cavity is still a mayor challenge. These two cases have produced by far the largest amount of differences in numerical results between procedures. Many authors 
have explained this effect as bifurcation of the solution. The idea of this work is to present a meshless localized RBF procedure to solve the flow equations in the original form, so that there is no simplification or approximation of any boundary condition. The velocity-pressure coupling procedure is the same one developed in [2]. The staggered point distribution approach (or grid) is selected and the RBF scheme is chosen to perform any necessary interpolation. Finally, in order to keep the numerical diffusion at a very low level, the well-known fluxlimiting scheme will be used in the discretization of the convection term.

\section{Velocity-pressure coupling}

The finite volume method proposed by Patankar, with SIMPLE and SIMPLER techniques as velocity-pressure coupling procedures, is the most popular method in CFD. These coupling schemes are used in most commercial and noncommercial CFD packages, using finite volume, finite difference or finite element method as the main discretization procedures. However, these coupling procedures are known to produce significant numerical diffusion. The most general procedure (SIMPLER) can be summarized as:

1. Discretize momentum equation $(\hat{p}=p / \rho-\vec{r} \cdot \vec{g})$ :

$$
\overrightarrow{\hat{v}}_{P}=\frac{1}{a_{P}} \sum a_{n b} \vec{v}_{n b}, \vec{v}_{P}=-\overrightarrow{\hat{v}}_{P}+\frac{1}{a_{P}} \nabla \hat{p}
$$

2. Compute pressure by introducing Eq. (1) into continuity equation:

$$
\nabla \cdot\left(\frac{1}{a_{P}} \nabla \hat{p}\right)=\nabla \cdot \overrightarrow{\hat{v}}_{P}
$$

3. Update pressure in Eq. (1) and solve for velocity.

4. Correct velocity to enforce mass continuity:

$$
\begin{gathered}
\vec{v}=\vec{v}^{*}+\vec{v}^{\prime}, \hat{p}=\hat{p}^{*}+\hat{p}^{\prime} \\
\nabla \cdot\left(\frac{1}{a_{P}} \nabla \hat{p}^{\prime}\right)=-\nabla \cdot \vec{v}^{*}+\nabla \cdot \overrightarrow{\hat{v}}^{\prime}
\end{gathered}
$$

where the term $\nabla \cdot \overrightarrow{\hat{v}}^{\prime}$ is frequently neglected. The first comment that it is convenient to make to this procedure is that, the main coefficient $a_{P}$ is inside all partial derivatives in pressure and mass-correction equations. The structure of this coefficient is:

$$
a_{P}=v C_{L}-u_{P} C_{D X}-v_{P} C_{D Y}
$$

with $C_{L}, C_{D X}$ and $C_{D Y}$ the coefficients of the discretization scheme. For a nonuniform mesh, the coefficient $a_{P}$ is a function of the position. This coefficient will produce clearly numerical diffusion in Eqs. (1) and (2). The only way that Eqs. (14) and (16) will not produce numerical diffusion is with a mesh of constant spacing and using central differencing in convection terms. Since $a_{P}$ gathers the diffusion and convection terms, central differencing for convection 
derivative will not have any coefficient. With a uniform mesh, the diffusion term of $\overrightarrow{\hat{v}}$ is zero and the Eq. (4) becomes exactly the same projection procedure of Harlow and Welch [1]. By updating velocity in SIMPLER, the procedure becomes the same algorithm [1], but in SIMPLE, updating pressure with $p^{\prime}$ is updating pressure with the velocity potential. This explains why SIMPLE takes so many iterations to converge and why this procedure works only when velocity is corrected and not pressure (as initially inferred).

Another problem that SIMPLE and SIMPLER have is, in pressure equation (4), boundary conditions (pressure coefficient zero in all boundaries) imply that the viscosity of the fluid is infinite at the wall, inflows and outflows. The condition of viscosity infinite is correct at the wall but, at inflows and outflows is evidently incorrect. At inflows, this change in the value of the viscosity of the fluid produces a force that helps the motion of the fluid but, at outflows, this change in viscosity produces a force that decelerates the fluid. This is one reason why pressure equation (4) has problems converging, unless a block-correction or multigrid algorithm is used. A useful alternative that solves the problems associated to both segregated and direct full coupling procedures is presented in [12]. In general, this scheme uses the segregated grid arrangement in the same way as finite volume method. The fundamental aspect of this coupling approach is that the velocity-pressure coupling is done with the momentum and continuity equations in the original form. After substituting finite differencing expressions for both, viscous and convection terms, as well as the pressure gradient, the momentum and continuity equations can be written in the form:

$$
\begin{gathered}
a_{P}^{u} u_{P}+C_{P}^{u}\left(p_{P}-p_{E}\right)=b_{P}^{u} \\
a_{P}^{v} v_{P}+C_{P}^{v}\left(p_{P}-p_{N}\right)=b_{P}^{v} \\
D_{P}^{u}\left(u_{P}-u_{W}\right)+D_{P}^{v}\left(v_{P}-v_{S}\right)=0
\end{gathered}
$$

In the system (6), pressure and velocity components are located at different points, as expected in a staggered point distribution. The coupling of $u, v$ and $p$ is performed by writing, in all possible ways, a linear system of 3 equations of the kind:

$$
\left(\begin{array}{ccc}
a_{11} & 0 & a_{13} \\
0 & a_{22} & a_{23} \\
a_{31} & a_{32} & 0
\end{array}\right)\left(\begin{array}{l}
u \\
v \\
p
\end{array}\right)=\left(\begin{array}{l}
b_{1} \\
b_{2} \\
b_{3}
\end{array}\right)
$$

whose solution is:

$$
\left(\begin{array}{l}
u \\
v \\
p
\end{array}\right)=\left(\begin{array}{c}
\frac{a_{23} a_{32} b_{1}-a_{13} a_{32} b_{2}+a_{13} a_{22} b_{3}}{a_{11} a_{23} a_{32}+a_{31} a_{13} a_{22}} \\
\frac{-a_{23} a_{31} b_{1}+a_{13} a_{31} b_{2}+a_{11} a_{23} b_{3}}{a_{11} a_{23} a_{32}+a_{31} a_{13} a_{22}} \\
\frac{a_{22} a_{31} b_{1}+a_{11} a_{32} b_{2}-a_{11} a_{22} b_{3}}{a_{11} a_{23} a_{32}+a_{31} a_{13} a_{22}}
\end{array}\right)
$$


Having in mind the linear system (6) and its solution (7)-(8), the discretized system (6) may be rearranged in many different ways. The solution procedure presented in [2] re-writes Eq. (6) in all possible permutations of both velocity components adjacent to pressure (see [2] for full details). The big advantage of this coupling procedure is that little memory is required to solve the corresponding linear system and that there are no errors in the boundary conditions. For the specific case of pressure, the problem is just an Initial Value Problem (IVP), and additionally in incompressible flows, only a reference for pressure is needed.

\section{Additional numerical schemes}

The velocity-pressure coupling alone is obviously not the only numerical issue to be solved in any fluid flow simulation. There are some other aspects where a lot of care must be taken in order to have a robust numerical procedure.

\subsection{Convection discretization}

Since the introduction of the first numerical procedures for CFD, the selection of a suitable discretization procedure for convection terms has not been an easy task. If the diffusion terms are going to be approximated with a second order finite differencing scheme, it makes sense to use a similar approach for convection terms and pressure gradient so that the whole scheme is second order.

It is well known that central schemes suffer from instability and that any upwinding approach is quite desirable, at the cost of some numerical diffusion. Since the procedure presented here is intended to be used in normal engineering calculations, as well as in DNS and LES simulations, the error in the convection term discretization must be kept as low as possible. One alternative, quite popular in the solution of the Euler equations and in the simulation of compressible flows is the idea of flux limiters. With all these considerations, in this work, the convection term will be discretized with the Osher flux limiter [23], with $\beta=2$, and using RBF to interpolate both velocity components at the corresponding interfaces.

\subsection{Interpolation}

Independent of the location of the variables in the grid, interpolation is a mandatory procedure. For example, collocated grid arrangement (all variables located at the same place) needs interpolation for the mass balance equation. Here the bilinear interpolation produces nonphysical oscillations and some nonlinear interpolation schemes have been proposed with great success, as for example [24]. On the other hand, the segregated approach, where velocity components and pressure are located at different positions and interpolation for the convection terms is needed. In this case, bilinear interpolation has been widely used since is stable and provides fast convergence. For example, in [25] and [26] a Radial-Based Function (RBF) procedure is chosen to perform all 
interpolations. In this procedure, $\mathrm{RBF}$ is used basically to compute the coefficients of all derivatives without the use of a connectivity mesh. No matter if this localized RBF collocation procedure is used to compute interpolations or derivatives, the main idea is that any field $\phi$ is represented by multiplying the basis functions by a set of expansion coefficients where, for stability reasons, a series of NP polynomial functions $P_{k}(\vec{x})$ are added to the interpolation scheme:

$$
\phi(\vec{x})=\sum_{j=1}^{N F} \alpha_{j} \chi_{j}(\vec{x})+\sum_{k=1}^{N P} \alpha_{k+N F} P_{k}(\vec{x})
$$

With respect to the basis function, the most accurate and stable one belongs to the group of Inverse Hardy Multiquadrics, which is of the form:

$$
\chi_{j}(\vec{x})=\frac{1}{\sqrt{r_{j}(\vec{x})^{2}+c^{2}}}
$$

where $r_{j}(\vec{x})$ is the Euclidean distance. At this moment, there is no unique way to determine the parameter $c$ so different formulations are employed. The expansion coefficients are then computed by writing Eq. (9) in every neighboring point and solving the corresponding linear system:

$$
[G] \vec{\alpha}=\vec{\phi}
$$

By substituting (11) in (9), a general interpolation scheme is obtained:

$$
\phi(\vec{x})=\sum_{i=1}^{N F}\left[\chi_{i}(\vec{x})\left(\sum_{j=1}^{N F} G_{i, j} \phi_{j}\right)\right]
$$

This Expression can be written as:

$$
\phi=\sum_{i=1}^{N F} b_{i} \phi_{i}
$$

The calculation procedure (9)-(12) must be preceded by a proper selection of the free parameter $c$. Practice has showed that the best results are obtained when the condition number of the matrix $G$ in Eq. (11) is between $10^{10}$ and $10^{12}$.

\section{Proposed algorithm}

As mentioned earlier, the method proposed here is a combination of several techniques found in FVM, FEM and meshless. The complete discretization procedure is: 
- Use a segregated grid arrangement for velocity components and pressure;

- Discretize the diffusion term with second order finite differences;

- Discretize the convection term with the Osher flux limiting scheme;

- Use RBF to interpolate velocity components (needed in the flux limiting scheme);

- Use second order central differencing for pressure gradient;

- Use second order central differencing for continuity equation;

- Solve the resulting system with the procedure explained in [8].

For the case of $2 \mathrm{D}$ steady flows, the main solution sequence proposed is:

1. Set an initial guess for velocity and pressure;

2. Set inflow and wall boundary conditions;

3. Compute diffusion coefficients in momentum equation and store them;

4. Compute pressure gradient coefficients and store them;

5. Compute coefficients in continuity equation and store them;

6. Store current velocity components;

7. Update velocity in outflow;

8. Compute momentum equation coefficients;

9. Perform block-corrections;

10. Interpolate velocity to the other grids;

11. Do some sub-iterations:

11.1 Update main variables from flow equations;

11.2 Adjust pressure of reference (to reduce numerical errors);

11.3 Find maximum increment.

If solution has not converged, go to step 6 .

\section{Laminar flow in a 2D backward-facing step}

The backward-facing step is one of the hardest validation cases since the structure of the flow is highly elliptic and it is excellent to show the robustness of any numerical procedure. This flow is characterized by a straight entry length, where the flow may or may not reach a fully developed state. Later the fluid enters in a sudden expansion and a big vortex is formed. In this zone, the structure is completely elliptic. Finally, the flow re-attaches and the parabolic regime is back again. In general, at low Reynolds numbers, the re-attachment increases and, when the flow enters in the transition stage, the re-circulation zone reduces to increase in size later as the speed of the flow increases.

In general, there is a very important disagreement between experiments and numerical predictions when the fluid enters in the transitional regime. Under very few exceptions, almost all numerical calculations fail to predict transition.

In [3] a time-dependent SIMPLE scheme is used and different transient procedures are tested. Calculations with Reynolds number of 800 are performed with an inflow length of 40 . Re-attachment occurs at $x=9.55$ after 50 seconds. A 
similar case can be seen in [4] where calculations are done with Reynolds of 800 and an inlet of $x=5$. A study is done by modifying the expansion ratio but no differences are reported with the entry length. A different approach is used in [5], where the boundary element method is used with primitive variables and an entry length of 0.02 is set. Validations are presented at Reynolds number of 500 but no comparisons are presented. Similarly in [6], a spectral method with primitive variables is used and calculations with Reynolds number of 800 are performed. Re-attachment is consistent with other references but no information of the location of the inlet. References [7] and [8] are similar cases where only one Reynolds number is used, 800. A study of outflow length is performed and the re-attachment is found to be consistent with previous works.

Some incomplete works can be found in [9] and [10]. In the first case, calculations with open and enclosed facing-step are performed but no comparisons are done on the re-attachment. In [10], SIMPLE scheme is used to perform 3D calculations with heat transfer but no information of inflow location is supplied. Here, some patterns are shown but no information on the reattachment.

In the literature, very few experiments have been published. The classical reference [11] is a complete study of the behavior of the flow in the whole laminar regime. The expansion rate used is 1.94 and the entrance length equal to $\mathrm{x}=40$, claimed to be enough to obtain fully developed flow at all Reynolds. Measurements show clearly a 3D pattern. The re-attachment shows a permanent increment up to Reynolds number to 1,200 , but then the size of the vortex decreases. Additionally, numerical calculations are performed using the measured inflow velocity profile and SIMPLE procedure. These calculations show an increment up to Reynolds of 450-500 and then decreases. This is the only numerical prediction that shows a decrement in the size of the vortex. Another series of experiments can be found in [12]. The geometry and conditions are the same as [11] and the results are in good agreement. Here, transition is measured at Reynolds number of 1,200 when the re-attachment decreases.

Several other works have been published where the Reynolds number used ranges up to 1,000. For example, in [13] an expansion ratio of 2 is used and the entrance length is set to 1 and the Reynolds number ranges from 150 up to 1050 . Re-attachment increases permanently. Stability analyses show that for Re from 750 and over, the flow becomes unstable to any 3D instability. Their results suggest that the flow is stable up to 1050 but they have not been able to compute for larger Reynolds. In [14], different entrance locations are tested. SIMPLE method with QUICK convection scheme is proposed and extrapolation for outflow boundary conditions is used. Calculations are done up to Reynolds of 800 and the re-attachment increases always. If no entrance, re-attachment increases tremendously. Similarly, in [15], computations are performed up to Reynolds of 800 with an expansion ratio of 1.94. In this paper, different convection schemes are tested, including TVD. All re-attachments increase as Reynolds except for the hybrid central-upwind that shows a decrement after Reynolds of 500. When refining the grid, all methods get an increasing Reattachment. Using an expansion ratio of 1.94 and Reynolds from 100 to 1,000 
[16], two different entry lengths are used, 0 and 10. In all cases re-attachment always increases. In [17], and expansion ratio of 1.94 is used with an entrance length of 2. Streamline-vorticity formulation is proposed and they consider that for $R e>1200$ the flow is transitional. For Reynolds equal to 800 , results agree with one reference but there is no detail for calculation with Reynolds larger than 1000 , only graphics at different scales. Reference [18] is perhaps the most complete calculation using the streamline-vorticity formulation. Some validations are presented with an expansion ratio of 1.94. It presents full results with expansion ratio of 2 and an inflow length of 20 . Reynolds number is varied from 100 to 3,000. Re-attachment always grows. No mention of transition and claims very accurate results. After Reynolds of 500, results differ from experiments. No mention that for higher Re, re-attachment decreases. Claim that re-attachment increases linearly as Reynolds increases. A study using primitive variables and Reynolds from 800 to 1,600 can be seen in [19]. Validation is presented for Reynolds of 800 and Hopf bifurcations are searched. Critical Reynolds is close to 1,200 . In [20], a streamline-vorticity Boundary Element Method is presented. No entrance region is set and Reynolds numbers are computed from 100 to 50,000. Mesh used is too coarse for the results to be correct. For Reynolds equal to 100 , re-attachment is very low compared to other calculations. For $R e=800$ good agreement is found but, for $R e=1,000$, no comparison is done. On the other hand, in [21], primitive variables and SIMPLE is used. Study effect of step height is done. Expansion ratio from 1.25 to 1.75 is analyzed and Reynolds number computed from 50-900. Good agreement with available data. In [22], a curvilinear coordinate system with QUICK scheme is proposed, with primitive variables and fractional step method. No entry length is used and the inflow profile is parabolic. Calculations are done with Reynolds up to 800 and re-attachment always increases. A different work can be found in [23], where a 3D simulation is done. The expansion ratio is 1.94 and the entrance region is equal to 1 . Numerical predictions are performed for Reynolds from 100 to 800 . Re-attachment increases always. One stability analysis through 2D DNS simulations can be seen in [24], where calculations are done for Reynolds number of 500 and 800 with different grid sizes. A very interesting study can be found in [25], where a streamline-vorticity formulation is used. The geometry is a sudden expansion, which can be seen as a double backward-facing step. For Reynolds number of 550, an asymmetric solution is obtained and for Reynolds of 786 , three different solutions are found, two of them asymmetric. There are some other references related to the backward-facing step, as for example [26] where a streamline-vorticity method is used, or [27] where a SIMPLE-based method is used. A transient study can be found in [28], where at the beginning, two separate vortices are formed, but after some time, they unify into one large vortex. In other works, as for example [29], several calculations with a Reynolds number from 10 to 610 are done, obtaining good agreement with experimental data. In a similar study [30], a calculation with a Reynolds number of 800 is done and good agreement is presented with available data. An old reference but very interesting is [31], being one of the first to report that the re-attachment is a function of the Reynolds number for laminar, but constant in turbulent regime. 


\section{Results and discussion}

Due to the large difference between calculations and experiments, we decided to use exactly the same geometry than in [11]. An expansion ratio of 1.94 was used, an inlet length of 38.4 and an outlet length of 100. At the inlet, a power profile was set in order to have a realistic boundary layer:

$$
\frac{u}{\bar{u}}=[1-|s|]^{1 / 100} ;-1 \leq s \leq+1
$$

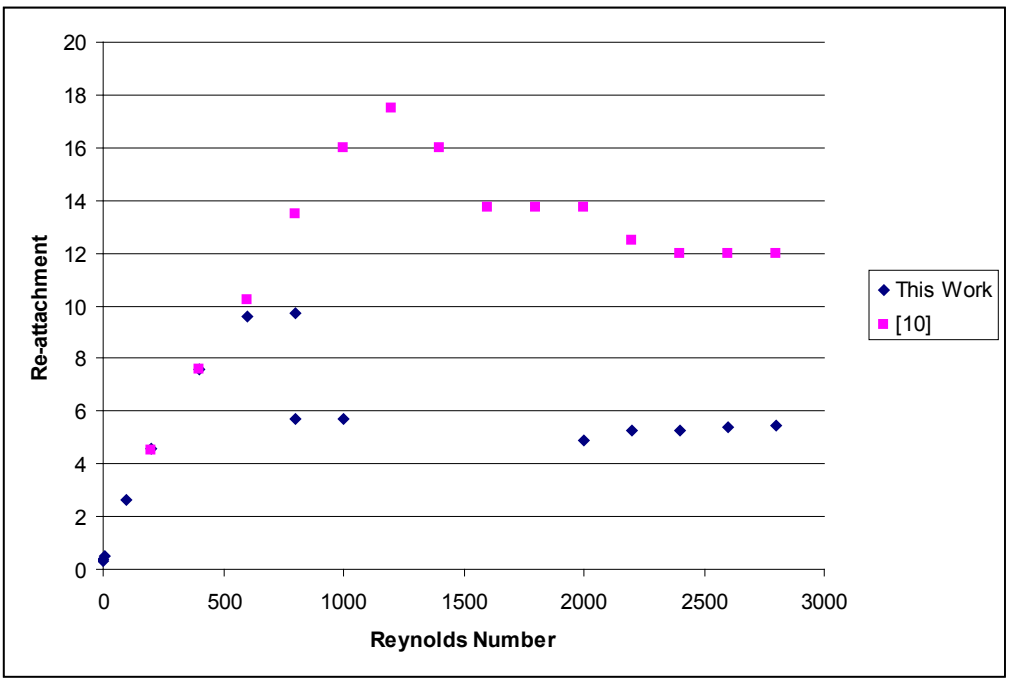

Figure 1: $\quad$ Re-attachment for an expansion ratio of 1.94.

A total of 288,208 pressure points were used, with an aspect ratio of $4: 1$. The calculations took about 2-3 hours for the lowest Reynolds and about 95 hours for Reynolds higher than 2,000. Figure 1 shows the comparison of the re-attachment of this work and the experimental measurements of Armaly [11]. At low Reynolds numbers, when viscous effects are dominant, the agreement is excellent. When the speed of the flow reaches Reynolds 800, the calculations became unstable and different solutions were found. For that Reynolds number, if the solution for 600 was used, the re-attachment was slightly higher but, when the solution for 1,000 was used, a low vortex was obtained. A similar situation was observed for the case of $1,200,1,400,1,600$ and 1,800. This phenomenon can be explained because at Reynolds 800 , inertia terms become important and they may, or may not, cancel the viscous terms, producing several solutions depending on convergence parameters. Once the speed of the flow is increased, inertia forces become dominant and basically one solution is obtained, as it was observed for Reynolds of 2,000 and over. 
It is important to underline that, for Reynolds over 600 , our results are underpredicted but the transitional state is correctly described. None of the references we reviewed, with the exception of the calculations of [11], show this behavior. We have to recall that all those studies show a permanent increment in the size of the re-attachment.

\section{Conclusions}

A meshless approach for incompressible fluid flow calculations has been proposed with the flow equations solved in strong form. The transitional regime, decrement of the re-attachment is correctly described.

\section{References}

[1] Harlow, F. H. and Welch, F. E., Numerical Calculation of Time-Dependent Viscous Incompressible Flow of Fluid with Free Surface, Physics of Fluids, 3, pp. 2182-2189, 1965.

[2] Vidal, A. and Rodríguez, J., A Direct Pressure-Linking Method for Turbulent Flow Computations, Numerical Heat Transfer, Part B, 52, pp. 531-549, 2007.

[3] Barton, I. E., Improved laminar predictions using a stabilized time dependent simple scheme, International Journal for Numerical Methods in Fluids, 28, pp. 841-857, 1998.

[4] Biswas, G., Breuer, M. and Durst, F., Backward-Facing Step Flow for Various Expansion Ratios at Low and Moderate Reynolds Numbers, Journal of Fluids Engineering, 126, pp. 362-374, 2004.

[5] Grigoriev, M. M. and Dargush, G. F., A poly-region boundary element method for incompressible viscous fluid flows, International Journal for Numerical Methods in Engineering, 46, pp. 1127-1158, 1999.

[6] Keskar, J. and Lyn, D. A., Computations of a laminar backward-facing step flow at $\mathrm{Re}=800$ with a spectral domain decomposition method, Intl. Journal for Numerical Methods in Fluids, 29, pp. 411-427, 1999.

[7] Sheu, T. W. H. and Tsai, S. F., Consistent Petrov Galerkin finite element simulation of channel flows, International Journal for Numerical Methods in Fluids, 31, pp. 1297-1310, 1999.

[8] Wagner, C., Hüttl, J. T. and Friedrich, R., Low-Reynolds number effects from direct numerical simulations of turbulent pipe flow, Computers \& Fluids, 30, pp. 581-590, 2001.

[9] Barton, I. E., Laminar flow past an enclosed and open backward-facing step, Physics of Fluids, 12, pp. 4054-4056, 1994.

[10] Nie, J. H. and Armaly, B. F., Three-dimensional convective flow adjacent to backward-facing step-effects of step height, International Journal of Heat and Mass Transfer, 45, pp. 2431-2438, 2002.

[11] Armaly, B. F., Durst, F., Pereira, J. C. F. and Schönung, B., Experimental and theoretical investigation of backward-facing step flow, Journal of Fluid Mechanics, 127, pp. 473-496, 1983. 
[12] Lee, T. and Mateescu, D., Experimental and numerical investigation of 2D backward-facing step flow, Journal of Fluid and Structures, 12, pp. 703716, 1998.

[13] Barkley, D., Gomes, M. G. M. and Henderson, R. D., Three-dimensional instability in flow over a backward-facing step, Journal of Fluid Mechanics, 473, pp. 167-190, 2002.

[14] Barton, I. E., The entrance effect of laminar flow over a backward-facing step geometry, International Journal for Numerical Methods in Fluids, 25, pp. 633-644, 1997.

[15] Biagioli, F., Calculation of laminar flows with second-order schemes and collocated variable arrangement, International Journal for Numerical Methods in Fluids, 26, pp. 887-905, 1998.

[16] Chiang, T. P. and Sheu, T. W. H., A numerical revisit of backward-facing step flow problem, Physics of Fluids, 11, pp. 862-874, 1999.

[17] Cruchaga, M. A., A study of the backward-facing step problem using a generalized streamline formulation, Communications of Numerical Methods in Engineering, 14, pp. 697-708, 1998.

[18] Erturk, E., Numerical solutions of 2-D steady incompressible flow over a backward-facing step, Part I: High Reynolds number solutions, Computers \& Fluids, 37, pp. 633-655, 2008.

[19] Fortin, A., Jardak, M., Gervais, J. J. and Pierre, R., Localization of Hopf bifurcations in fluid flow problems, International Journal for Numerical Methods in Fluids, 24, pp. 1185-1210, 1997.

[20] Ramsak, M. and Skerget, L., A subdomain boundary element method for high-Reynolds laminar flow using stream function-vorticity formulation, International Journal for Numerical Methods in Fluids, 46, pp. 815-847, 2004.

[21] Thangam, S. and Knight, D. D., Effect of stepheight on the separated flow past a backward facing step, Physics of Fluids A, 1, pp. 604-606, 1989.

[22] Zang, Y., Street, R. L. and Koseff, J. R., Grid A Non-staggered Fractional step method for time-dependent incompressible Navier-Stokes equations in curvilinear coordinates, Journal of Computational Physics, 114, pp. 18-33, 1994.

[23] Le, H., Moin, P. and Kim, J., Direct numerical simulation of turbulent flow over a backward-facing step, Journal of Fluid Mechanics, 330, pp. 349374, 1997.

[24] Yee, H. C., Torczynski, J. R., Morton, S. A., Visbal, M. R. and Sweby, P. $\mathrm{K}$., On spurious behavior of CFD simulations, International Journal for Numerical Methods in Fluids, 30, pp. 675-711, 1999.

[25] Alleborn, N., Nandakumar, K., Raszillier, H. and Durst, F., Further contributions on the two-dimensional flow in a sudden expansion, Journal of Fluid Mechanics, 330, pp. 169-188, 1997.

[26] Coates, M. J. and Patterson, J. C., Numerical simulations of the natural convection in a cavity with nonuniform internal sources, International Journal of Heat and Fluid Flow, 15, pp. 218-225, 1994. 
[27] Demirdzi, I. and Peric, M., Finite-volume method for prediction of fluid flow in arbitrary shaped domains with moving boundaries, International Journal for Numerical Methods in Fluids, 10, pp. 771-790, 1990.

[28] Durst, F. and Pereira, J. C. F., Time-dependent laminar backward-facing step flow in a two dimensional duct, Journal of Fluids Engineering, 110, pp. 289-296, 1988.

[29] Durst, F., Pereira, J. C. F. and Tropea, C., The plane symmetric suddenexpansion flow at low Reynolds numbers, Journal of Fluid Mechanics, 248, pp. 567-581, 1993.

[30] Gartling, D. K., A test problem for outflow boundary conditions-flow over a backward-facing step, International Journal for Numerical Methods in Fluids, 11, pp. 953-967, 1990.

[31] Goldstein, R. J., Eriksen, V. L., Olson, R. M. and Eckert, E. R. G., Laminar separation, reattachment, and transition of the flow over a downstreamfacing step, Journal of Basic Engineering, 92, pp. 732-739, 1970. 